\title{
DEMOCRACIA Y ECOLOGÍA. UN ANÁLISIS DE LAS CONDICIONES DE SOSTENIBILIDAD EN EL CAPITALISMO DE ALTA INTENSIDAD
}

\author{
DEMOCRACY AND ECOLOGY. ANALYSIS OF THE CONDITIONS \\ OF SUSTAINABILITY IN HIGH INTENSITY CAPITALISM
}

Rafael Rodríguez Prieto

Recibido: julio de 2011

Universidad Pablo de Olavide de Sevilla Aceptado: septiembre de 2011

E.mail.: rrodpri@upo.es

Palabras clave: ecología, sostenibilidad, democracia, producción.

Keywords: ecology, sustainability, democracy, production.

Resumen: Las interacciones actuales y futuras entre seres humanos y sistemas naturales impactarán significativamente sobre la sociedad y el medio ambiente. Estos temas centrales precisan de ser comprendidos e investigados conectados con la sostenibilidad, desarrollando nuevas aproximaciones al modo como nos relacionamos con el entorno, el uso de la tierra, el desarrollo y la industria. Comprender la verdadera naturaleza de una sociedad sostenible con el medio ambiente es una cuestión compleja a la que los investigadores están prestando atención desde disciplinas diversas. Este artículo es crítico en un mundo en que los recursos fósiles no renovables son la principal fuente de energía en detrimento de ecosistemas naturales. La protección medioambiental debería buscar reemplazar una perspectiva estrecha por una acción democrática y políticas participativas que posibiliten un bineestar común. La sostenibilidad materialistas es una alternativa a la aproximación hegemónica ala naturaleza. Necesitamos desarrollar nuevas estrategias para producir bienes y servicios desde un punto de vista sostenible.

Abstract: Current and future interaction between humans and natural systems will impact significantly on society and the environment. This highlights the need to understand and research the nature of sustainability and develop new approaches to lifestyle, land use, development and industry. Understanding the true nature of a sustainable society and environment is a complex question that scholars are addressing across many sectors and disciplines.My paper is critical in a world that relies on non-renewable resources, such as fossil 
fuels, and puts increasing strain on ecosystems and natural resources. The environmental protection must seek to replace a narrow perspective with democratic action and cooperative participatory politics for common stewardship. Materialist sustainability is an alternative to the hegemonical approach to nature. We need to develop new strategies in order to produce goods and services in a sustainable way.

«Toda la población actual, agrupada en familias de cuatro personas que tendrían 500 metros cuadrados de tierra cada una cabrían prácticamente en el Estado de Tejas [696.241 kilómetros cuadrados]. El problema no es de espacio, sino de cómo tratamos el medio ambiente. Los recursos se han manejado sin criterios de eficiencia. Generamos una cantidad de basura escandalosa. No reciclamos. Se destruyen alimentos para preservar los precios...».

Julio Pérez ${ }^{1}$

\section{Introducción}

Con motivo del nacimiento del habitante 7000 millones los medios de comunicación prestaron una atención especial al equilibrio entre los recursos y el crecimiento de la población en nuestro planeta. Asia es el continente en donde se produce un crecimiento mayor de la población, a la vez que las condiciones de sostenibilidad se degradan de forma más evidente. Sostenibilidad ambiental, pero también las condiciones que afectan a la diferencia entre pobres y ricos. Quizá el país que represente de forma más aguda esta situación sea China.

China ha emergido en los últimos años que el nuevo hegemón en el campo de las relaciones internacionales. China posee gran parte de la deuda de EE.UU. y la UE está pugnando porque coopere aún más en la crisis de deuda por la que atraviesa. La influencia creciente del país asiático en la arena global ha hecho que una nutrida parte de estudiosos y experto vuelvan su mirada hacia él. ¿Puede mantener China su exponencial crecimiento, a la vez que resuelve las tensiones internas? ¿Será China el nuevo jefe tras el declive de EE.UU?

Quizá de los riesgos mayores que tiene China que afrontar sean las tensiones internas. El crecimiento ha disparado las diferencias sociales y estas desigualdades podrían ser un foco de problemas futuros, a pesar del fuerte Estado policial chino. Supongamos que el gobierno quiere evitarlo. El resultado sería tratar de mejorar las condiciones de vida de la mayoría de la población. Tienen recursos para ello. El problema es que según los expertos es imposible que nuestro planeta pudiera aguantar la promesa consumista del capitalismo actual. La sostenibilidad quedaría aún más en entredicho, siendo la mejora en la cali- 
dad de vida de las personas, patrimonio exclusivo de unas pocas personas. China representa como pocos los grandes dilemas del modelo productivo actual.

¿Cómo conciliar crecimiento con sostenibilidad y cuidado del medio ambiente? ¿Cómo inciden los modelos productivos en ello? ¿Cuál es el papel de la democracia y de los ciudadanos? ¿Es la crisis ecológica parte de la crisis económica?

La apelación al desarrollo sostenible se ha convertido en un lugar común en los últimas décadas. Desde que en 1987 el Informe Brundtland lo definiera como aquel que satisface las necesidades del presente sin comprometer a las futuras generaciones, se ha convertido en un lugar común de ONGs, gobiernos y otros colectivos e instituciones. Se trata de un informe amplio y complejo en el que podemos subrayar dos elementos fundamentales: la idea de conservación del planeta y la necesidad de renunciar a los niveles de consumo de 1987.

Después de casi un cuarto de siglo la realidad se torna preocupante. La protección de la Tierra y de los ecosistemas ha mejorado levemente o no ha sido tan destructiva en algunos Estados, especialmente los pertenecientes a la UE y ciertas áreas de América (EE.UU., Canadá o Costa Rica). Sin embargo, los abusos sobre los ecosistemas continúan en el resto del planeta y el fracaso en las Cumbres, que repetidamente se organizan en el marco de Naciones Unidas, se ha convertido en una costumbre que los gobiernos tratan de ocultar. La Cumbre del Clima de 2009 en Copenhague o la de Cancún de 2010 se quedaron en una mera declaración de intenciones, mientras que el Protocolo de Kyoto sigue siendo sistemáticamente incumplido y aún se desconoce si tendrá algún tipo de continuidad después de 2012. Tal y como señalan el grupo de expertos que avalan el Anuario económico geopolítico mundial «El estado del mundo 2011», la crisis ecológica derivada de estas tensiones entre Estados se ceba en las capas más pobres e indefensas de la población, que sufren los peores efectos del cambio climático traducidas en enfermedades y hambre?

Pero si la conservación arroja resultados decepcionantes, aún es peor en el caso de los niveles de consumo. La idea del crecimiento económico como sinónimo de prosperidad y la celeridad en la obsolescencia de los bienes está acrecentando el nivel de residuos que emitimos al medio ambiente. Schumacher fue el gran pionero de los estudios que ponían en cuestión el mito del crecimiento ilimitado y la relación entre el el PIB y la felicidad. Su idea de economía budista y su apelación la construcción de un orden en equilibrio con la naturaleza realizada en su libro seminal Lo pequeño es hermoso, fueron celebradas por los movimientos sociales de su época, con la misma intensidad que olvidadas por los gobernantes y agentes económi$\cos ^{3}$.

Cuando los problemas medioambientales se tornaron como evidentes, algunos Estados industrializados tomaron medidas en lo que respecta al procesamiento de residuos o a la protección de áreas de valor ecológico. No obstante, al mismo tiempo se multipli- 
caron exponencialmente los tipos de basura y el limitado el número de países con capacidad para procesarla.

El objetivo de este trabajo es bastante modesto. Cuando planteo la pregunta de si puede ser conciliable el capitalismo con la sostenibilidad estoy tratando de llegar al núcleo del nudo gordiano que desde 1987 tenemos planteado y que se repite una y otra vez en las diferentes cumbres. ¿Puede haber una considerable restricción en los niveles de consumo y en la protección de la naturaleza sin afectar a la esencia misma del capitalismo? ¿Es el capitalismo insostenible onto-lógicamente? Mi hipótesis es que si pretendemos garantizar la sostenibilidad tendremos que afectar a la propia dinámica capitalista. Habrá que decidir qué preferimos y abandonar consideraciones escasamente rigurosas e ideológicas que pretenden lograr la sostenibilidad sin afectar al modelo productivo y cultural capitalista.

Las instituciones, la política, la economía, el derecho e incluso la sociedad se adaptan al capitalismo hoy más que nunca en la historia de la humanidad. Es un capitalismo de alta intensidad que disciplina el resto de áreas de la vida. De lo contrario, «intranquilizan a los mercados» y su estabilidad puede verse en entredicho ${ }^{4}$. Como ha señalado el propio Jean Claude Trichet, Presidente del Banco Central Europeo, es necesaria una política fiscal única en la UE. Esta declaración de Trichet sitúa a la soberanía económica en manos de un poder tecnócrata, en vez de en los parlamentos ${ }^{5}$. Una de las consecuencias más relevantes de la crisis financiera, que acucia a las economías a escala global, ha sido el recorte de derechos sociales en Europa. En otras partes del mundo, donde jamás se ha gozado de tal redistribución, la situación de los trabajadores es precaria.

China es un polo de atracción para las corporaciones y ejemplo de país dominado por una oligarquía que controla policialmente el territorio. La exitosa marcha económica de China, que la ha convertido en la segunda potencia a nivel mundial, tiene un contrapunto en las divisiones en la renta que existen en ese país. No es descartable que se vivan tensiones futuras por ello. China además ha obtenido cierta notoriedad por su particular negativa a cualquier medida de protección medioambiental. De esa forma se ha convertido en la gran fábrica del mundo. Países como España han tenido que dar marcha atrás a sus ambiciosos programas de estímulo de energías alternativas, presionados por el objetivo de reducción del gasto público.

Esta apelación a la influencia radical de la economía en un artículo sobre sostenibilidad es muy pertinente. Si no contextualizamos el problema de la sostenibilidad medioambiental corremos el riesgo de empantanarnos en consideraciones que hagan abstracción de las condiciones materiales donde tienen lugar las acciones humanas que inciden sobre el equilibrio con la naturaleza. En este sentido, actualmente se está usando mucho el concepto de biomímesis consistente en imitar la naturaleza a la hora de reconstruir los sistemas productivos humanos, con el fin de hacerlos compatibles con la biosfera ${ }^{6}$. En este texto lo tomaremos como sinónimos. 
La degradación medioambiental crece sin parar. Según el estudio de la ONU de 2005 titulado «Evaluación de los Ecosistemas del Milenio», el deterioro encierra el riesgo de cambios potencialmente graves que tendrían una repercusión muy seria sobre el bienestar humano. Subraya que en los últimos 50 años, los seres humanos han modificado los ecosistemas más rápidamente que durante cualquier otro periodo de la historia. Más del $60 \%$ de los servicios de los ecosistemas que hacen posible la vida en la Tierra está siendo degradado o utilizado de manera no sostenible. Tal y como señalan sus redactores, «ni siquiera se puede decir que el producto de esta degradación progresiva suponga un beneficio para las personas». El Informe señala en su diagnóstico, que a pesar del progreso conseguido en el aumento de la producción, los niveles de pobreza siguen siendo altos y las desigualdades crecen ${ }^{7}$.

Pero tampoco se pueden ignorar la existencia de iniciativas que tratan de apostar por un crecimiento sostenible en las sociedades occidentales. Hemos citado el caso de las energías renovables, auspiciadas por Estados y empresas, pero también hay prácticas como el reciclaje o la producción ecológica de ciertos bienes relevantes para la sostenibilidad. Incluso existen corrientes de pensamiento favorables al decrecimiento. Grandes corporaciones se anuncian como ecológicamente responsables. Los gobiernos se reúnen periódicamente con el fin de limitar los efectos perniciosos de la industrialización. ¿No sería ésta una tercera vía que concilie sosteni-bilidad y capitalismo? De ello nos ocuparemos posteriormente.
El debate sobre la sostenibilidad se plantea en un contexto muy complicado. La preocupación por la naturaleza, desde la emergencia de los nuevos movimientos sociales preocupados por hacer un uso más responsable de los recursos naturales, ha ido en aumento. En países como Alemania, los ecologistas no sólo han ocupado espacio en los parlamentos, sino además han tenido responsabilidades de gobierno en coalición con los socialdemócratas. A la vez, se suceden las reflexiones y discusiones en torno al calentamiento global y otros retos ecológicos que afronta el planeta. Podemos distinguir tres grandes maneras de enfocar tales desafíos.

Una primera forma sería la hegemónica centrada en limitar los efectos perniciosos para la naturaleza de la actividad industrial. Esta vía se institucionaliza mediante las cumbres medios ambientales en que participan los Estados, la creación de ministerios y agencias medioambientales en los gobiernos y las medidas que toman las grandes corporaciones transnacionales. El desarrollo sostenible, avalado por Naciones Unidas o cumbres como las ya mencionadas de Cancún o Kioto forman parte de esta línea de actuación8.

Una segunda forma es la de pequeños y numerosos colectivos de activistas que proponen un consumo responsable que mejore las relaciones entre ser humano y naturaleza. Estos grupos critican tanto a gobiernos como a corporaciones transnacionales por su limitado compromiso medioambiental. Consideran que la sociedad civil tiene la responsabilidad de presionar y convencer a empresas y 
gobiernos para que rectifiquen su postura y cooperen activamente en la mejora del medio ambiente y extender una sostenibilidad real que garantice la vida de las generaciones futuras.

Junto con las anteriores, existe una tercera manera. Esta vía integra el problema medioambiental en la producción y en la conformación de un orden cultural, político y socioeconómico dominado por el capitalismo de alta intensidad. No se puede entender la cuestión ecológica aislada de otros problemas. Desde esta perspectiva, no se puede separar el debate sobre sostenibilidad del de los principios y valores que inciden sobre la misma. La economía neoclásica y sus principios no pueden escindirse, de forma ideológica e interesada, de la reflexión sobre la sostenibilidad y la protección del medioambiente. Es escasamente realista pensar que una idea aislada va a alterar la lógica y la dinámica del conjunto de valores, ideas y estrategias que gobiernan el mundo. Si queremos cambiar las cosas y lograr la sostenibilidad, debemos dirigirnos al corazón del problema a la vez que conectamos saberes y problemas con el fin de encontrar soluciones satisfactorias ${ }^{9}$.

En definitiva, podemos distinguir tres enfoques: uno presidido por la producción capitalista, donde la sostenibilidad es un objetivo lejano a perseguir, que decae en beneficio de la maximización del provecho económico; otro por la producción capitalista limitada, donde la sostenibilidad puede ser parte del negocio; y un tercero por la producción democrática o participativa, donde la sostenibilidad es parte de la propia dinámica productiva.
La problemática medioambiental está radicalmente imbricada en la perspectiva filosófica que asumamos y la visión que tengamos del valor y del trabajo. Siempre se suele hacer esta mención de la cultura o de la religión, pero no es menos cierto de la economía y del modelo de relaciones productivas que asumamos. El medio ambiente y su protección no puede ser concebido como un compartimento estanco sobre el que actuar de forma particular. Descartes dedujo que todo era dudoso menos el yo pienso, por lo que la propuesta idealista de concebir la sustancia como sujeto ${ }^{10}$ es una herencia de una comprensión mecanicista cartesiana del mundo. La física contemporánea está mostrando una visión holística y dinámica del universo ${ }^{11}$. Como señala Pérez Argote, "para dilucidar el significado social del medio ambiente es preciso observar todas las estructuras sociales, los procesos ${ }^{12}(\ldots)$ que hacen del medio ambiente un problema sociológicamente relevante. El medio ambiente es una cuestión de poder ${ }^{13}$.

Mi tesis es que la primera y segunda tienen insuficiencias que hacen inviable el mantenimiento de una sostenibilidad realista entre ser humano y naturaleza. La ideología medioambientalista o como ha sido denominado por otros autores, el ecoblanqueamiento ${ }^{14}$ trata la cuestión medioambiental como un problema particular que divorcia de un mega-problema amplio y complejo planteado por la producción y la cultura derivada del propio modelo productivo y de consumo.

Mi contribución será desarrollar el principio de sostenibilidad mediante una 
transformación del modelo productivo que lógicamente precisa de prácticas de democracia directa que constituyan una nueva eficiencia, a la que denomino ecológica, en el marco de una lógica de la responsabilidad. Para ello realizaré un estudio de las tres aproximaciones planteadas y propondré las líneas elementales de una aproximación materialista a la sostenibilidad. Como acertadamente señala Jorge Riechmann, ecologizar nuestros sistemas socioeconómicos exige pasar de esta civilización de la «sociedad del riesgo» a la «sociedad de la precaución». La solución no consiste en bloquear los conocimientos, sino en gobernarlos, evaluando las alternativas tecnológicas y cerrando el paso a las aplicaciones que conlleven riesgos demasiado altos ${ }^{15}$.

\section{La sostenibilidad en perspectiva: la producción capitalista y la producción capitalista limitada}

Sostenible o sostenibilidad es una de las palabras que han adquirido más relevancia y prestigio en los últimos años. Todo se pretende que sea sostenible. El desarrollo, la economía, el uso de la energía, etc., han tenido una notable trascendencia en reuniones internacionales o medios de comunicación. Como señalaba en el epígrafe anterior, sostenible es aquella característica o estado según el cual pueden satisfacerse las necesidades de la población actual sin comprometer a generaciones futuras. En resumen: el uso sostenible de un eco- sistema consiste en preservarlo para produzca beneficio continuo tanto para generaciones actuales como futuras. Si levantamos la vista de libros y definiciones, la realidad parece bastante diferente. No sólo por las agresiones que constantemente padece el medio ambiente en el que habitamos. Recordemos el publicitado vertido de petróleo en el Golfo de México, la mercantilización de los bosques rusos, la contaminación sistemática de ríos en Ecuador o el escaso acuerdo en el control de emisiones contaminantes. Pero también hay otros ejemplos que nos dan esperanza: empresas dedicadas a la potenciación de energías renovables o de construcción ecológica y cumbres internacionales en las que se alcanzan acuerdos para restringir las emisiones de gases de efecto invernadero.

Hace unos años se publicó en el mundo un libro muy controvertido titulado $E$ I Ecologista Escéptico. En ese trabajo B. Lomborg -un antiguo activista- planteaba una crítica radical a la tesis ecologista mayoritaria consistente en que la situación medioambiental es cada vez peor. Para Lomborg, un profesor de estadística, los hechos desmienten esta idea. Sostiene que un debate tan importante como el del medioambiente «se base más en mitos que en realidades» ${ }^{16}$.

Lomborg se ha retractado en parte de sus posiciones tras reconocer en 2009 la necesidad de que se invierta para evitar el calentamiento global. Aunque coincido con Nature en las críticas a la posición del autor noruego por su optimismo infundado ${ }^{17}$, no puedo dejar de admitir que tiene razón cuando afirma 
que el desarrollo tecnológico en los últimos decenios podría presagiar que se dan las posibilidades para evitar el calentamiento global y los problemas medioambientales más relevantes que afrontamos.

El avance tecnológico experimentado en las últimas décadas pone de manifiesto que la sostenibilidad es viable y posible. Sin embargo, los datos apuntados por activistas y científicos que se ocupan de los progresos en protección medioambiental indican lo contrario. Esta carencia de sostenibilidad ha sido percibida por la ciudadanía de algunos lugares y ha creado un malestar ecológico, que se ha traducido en movimientos sociales, consumo responsable, activistas contra la agresión al medio ambiente o conferencias internacionales patrocinadas por la ONU, con el fin de garantizar una serie de derechos en esta materia. Incluso, parte del empresariado ha visto como una oportunidad de negocio el desarrollo de la producción con conciencia medioambiental.

Desde los años setenta, la cuestión medioambiental está en la agenda de colectivos ciudadanos, organizaciones internacionales y empresas. Si queremos ser realistas habrá que decir que el saldo no es muy positivo. La bibliografía sobre el tema es abrumadoramente pesimista. La falta de sostenibilidad no sólo decrece, sino se refuerza y aumenta. ¿Qué razón puede haber para ello?

Desde la perspectiva de la producción capitalista, la sostenibilidad es una preocupación, pero no se sitúa por encima de la obtención de beneficios. Con el fin de incentivar a las empresas a producir de manera más ecológica y con un mayor grado de sostenibilidad, sin afectar a la maximización del beneficio, se han creado mecanismos específicos. Entre estas líneas de actuación cabe mencionar la compra de emisiones de gases, las subvenciones que administraciones públicas ofrecen a empresas, nichos empresariales relacionados con energías verdes, etc.

¿Se pueden conciliar éticamente intereses tan aparentemente dispares como la ecología y la obtención de un beneficio ilimitado para los accionistas? ¿Cómo se inserta la problemática sobre el medio ambiente en la democracia? En este sentido resultan relevantes los trabajos de Dryzek, Sosa y Torgerson.

Para éste último, el ciudadano en la democracia liberal es concebido como individuo racional que actúa para realizar sus preferencias en un contexto competitivo determinado por una serie de reglas del juego. Este análisis fundado en el individualismo posesivo implica incentivos que sirven para formar y dirigir el comportamiento, que a su vez son estructurados por organizaciones formales que no son sólo instituciones estatales, sino también corporaciones cuyo impacto sobre políticas públicas y hábitos de consumo son muy profundos ${ }^{18}$. De esta forma, se abandona el medioambiente a su suerte y queda simplemente como un objeto que explotar. Este enfoque es consecuencia de un tipo de ética individualista y antiecológica.

El profesor español Nicolás Martín Sosa apuesta en sus escritos por una nueva ética ecológica que estime que las preferencias o intereses humanos no son 
preferentes. Los intereses comunes a humanos y no humanos serían los supremos. El sujeto moral que asume una ética ecológica habita no sólo una casa humana o social, sino una casa planetaria o e todos y es por ello que debe asumir una responsabilidad mayor ante los bienes de todos, siendo en este sentido fundamental la ética ecológica que proponga un cuestionamiento global de nuestras prácticas en el mundo que conduzca a una sociedad sostenible más allá del consumis$\mathrm{mo}^{19}$. El consumismo sitúa al ser humano ante un dualismo falso: crecer o estancarse y perecer.

Dryzek completa este debate estimando que el sistema de "crece o muere» hegemónico en las democracias capitalistas, postulado por autores como Fukuyama, es insostenible a la luz de los límites ecológicos a los que nos enfrentamos, cuyas manifestaciones son los problemas medioambientales que todos conocemos. La idea central de la democracia liberal como agregación de preferencias no es suficiente para romper la barrera con el medioambiente que condena cualquier acción en este sentido $^{20}$. La posibilidad de ajustar equilibradamente el medioambiente y los pilares del capitalismo es ética y democráticamente complicado. Sus lógicas parecen difícilmente conciliables, cuando no antagónicas.

La producción capitalista basa su dinamismo en el crecimiento económico. Los productos deben ser consumidos para que las economías crezcan. Un ejemplo de ello lo obtenemos en Estados como Alemania, que ha podido aban- donar la crisis antes que España porque han comenzado a crecer a un ritmo vertiginoso. La teoría del crecimiento económico consiste en una producción eterna de bienes y servicios. La idea general es que un país produzca bienes y servicios gracias a la inversión de capital. El producto de todo ello será la creación de puestos de trabajo. Del crecimiento se derivan recursos para aumentar la capacidad de consumo. Este modelo de producción tiene su talón de Aquiles en la necesidad de mantener también una fuerte demanda de productos; de lo contrario, el stock generaría problemas. Hace falta el consumismo. Es fundamental para que esta dinámica se mantenga.

Una tasa de crecimiento económico de un $2 \%$ anual, por ejemplo, significa que se produjeron un $2 \%$ más de bienes que el año anterior y un $2 \%$ menos que el año venidero. ¿Es posible convertir el medioambiente en bienes y servicios eternamente, como lo requiere esta teoría? Según Giles Slade, la obsolescencia de los productos puede ser planificada con el fin de posibilitar el crecimiento y el consumismo. En su libro Made to break, se ponen algunos templos históricos de ello. Aunque este hecho no es nuevo, Slade desarrolla gran parte de su argumento en relación con era de las computadoras. En este momento histórico, la tesis de la obsolescencia ha alcanzado sus más altas cotas de desarrollo y la basura que se genera de todo ello, su mayor número. En 2004, alrededor de 315 millones de PCs se reemplazaron por nuevos modelos ${ }^{21}$. Sólo el $10 \%$ fueron aprovechados, mientras que el resto se convirtieron en basura que 
se exporta a países empobrecidos de África. Hace siete años de este dato. Imaginemos el impacto que están teniendo otros bienes tecnológicos que son reemplazados con rapidez, como móviles, impresoras o i-pods y nos podremos hacer una idea de lo que sucede.

El problema es que la Naturaleza no puede ser eternamente transformada en bienes para el consumo porque sólo hay una cantidad de recursos disponibles. Seguir aferrados a la teoría del crecimiento económico es aceptar a una ficción, a una sostenibilidad ideal, pero poco conectada con las condiciones materiales del planeta y las personas que lo habitan. Por muchas iniciativas y nichos de negocio que se abran que sean responsables con el medioambiente, se abrirán otros muchos la dirección opuesta, especialmente en Estados empobrecidos de África, Asía o América, que compiten con Europa o EE.UU. en normativas muy flexibles o inexistentes de protección medioambiental. Pero es además la propia dinámica del crecimiento económico, la que condena cualquier atisbo de sostenibilidad en este modelo productivo. La obsolescencia programada, como hemos visto, es una consecuencia de la necesidad de consumo para que la producción no pare y se mantenga la dinámica del crecimiento.

No obstante, existen posturas que no renuncian a conciliar capitalismo con medio ambiente. Desde hace tiempo, han emergido grupos y prácticas que postulan tesis, experiencias y prácticas que desean una producción capitalista limitada que posibilite una sostenibilidad real de los recursos. Una de las ideas ha sido incentivar la compra en mercados cercanos que sean ecológicos y respetuosos con el medio ambiente. Impulsar las energías renovables, así como el comercio justo son otras prácticas que tratan de limitar los efectos nocivos anteriormente aludidos.

The Whole Earth Catalog es un perfecto representante de esta posición. Esta publicación amparó gran parte del movimiento contracultural y ecologista de Ios EE.UU. Sus posiciones sobre medioambiente eran pragmáticas y partidarias de los logros tecnológicos y la sostenibilidad. Alrededor de esta publicación se desarrollaron durante tres décadas (50-70) una serie de colectivos y movimientos que posibilitaron la difusión de sus contenidos y del debate sobre la sostenibilidad. El objetivo era reconciliar el capitalismo, la tecnología, la libertad, la seguridad y la naturaleza. La aceptación, por tanto, del modelo capitalista, era central en este esquema. Esta inequívoca voluntad de hacer sostenible al capitalismo con el medio ambiente fructificó en un auge de los negocios verdes que impulsó un modelo de negocio contracultural de gran éxito y con influencia en el auge de las empresas «punto com» en los noventa ${ }^{22}$.

El consumo verde y el deseo de alternativas respetuosas con el medio ambiente generó una dinámica que postulaba un cambio en las prácticas empresariales de EE.UU., sin que se tuviera que alterar lo esencial del modelo económico. Uno de los casos más famosos fue la presión ejercida por estos colectivos sobre McDonald para que sustituyera los plásticos (que generaban mu- 
cho CFC en su fabricación) por envoltorios de papel. Este tipo de consumo alternativo se basó en la acción política y representó una vía que aún continúa vigente en los EE.UU.

Paul Hawken estima que los hombres de negocio deben cambiar las bases éticas de su conducta y mejorará la relación entre capitalismo y medioambiente. El argumento de Hawken se centra en culpar a los individuos de los problemas medioambientales ${ }^{23}$. La cuestión decisiva es el individuo y su responsabilidad frente a la naturaleza. Se viene a decir que si el individuo es bueno e influye en los individuos que producen y consumen los productos de las empresas, el resultado será que se podrá conciliar el capitalismo con el medio ambiente.

El problema es que esto no es así. Los individuos, aún suponiendo la mejor predisposición hacia la conservación de la naturaleza, son rehenes de unos modelos productivo y de una dinámica cuyo único fin es preservar el mayor número de beneficios para los accionistas. No obstante, la relevancia de esta alternativa conciliadora es indudable. La idea de que se puede incorporar la sostenibilidad al medioambiente con éxito y, lo que es más, posibilitando crecimiento y desarrollo económico goza de un gran predicamento en el mundo académico general y en muchas escuelas de economía, en particular. El problema, sin embargo, es que los indicadores medioambientales a nivel global son cada vez peores, a la vez que el éxito de está perspectiva queda muy limitado a determinados Estados de Occidente con legislaciones medioambientales exigentes y con capacidad para incentivar un consumo responsable entre sus ciudadanos. El impacto de los productos verdes, justos o ecológicos es muy limitado en comparación con el resto ${ }^{24}$. La gente en situación de crisis demanda productos baratos, procedentes de marcas blancas o de la producción masiva que tiene su foco en China. La crisis no es una buena amiga de la sostenibilidad.

Estas posiciones conciliadoras han sido criticadas desde muy diversos ámbitos y posiciones. En los últimos años ha hecho fortuna en España y el resto de Europa una alternativa ecológica que postula el decrecimiento. Esta idea retoma informes de los años setenta, como el del Club de Roma, que ya por aquel entonces -1972- postulaban del crecimiento cero. El decrecimiento es una corriente de pensamiento político, económico y social favorable a la disminución controlada de la producción económica con el objetivo de establecer una nueva relación de equilibrio entre el ser humano y la naturaleza. Los partidarios del decrecimiento proponen una disminución del consumo (material y energético) y de la producción controlada y racional, permitiendo respetar el clima, los ecosistemas y los propios seres humanos. Esta transición se realizaría mediante la aplicación de principios más adecuados a una situación de recursos limitados: escala reducida, relocalización, eficiencia, cooperación, autoproducción (e intercambio), durabilidad y sobriedad $^{25}$. Según Taibo, la mitología del crecimiento se sustenta sobre la publicidad, el crédito y la caducidad. Esta mitología no garantiza que con el crecimiento van a incrementarse los niveles de bienes- 
tar. Al contrario, con crecimiento, la cohesión social o las agresiones medioambientales, por poner dos de los ejemplos más destacados, se pueden ver incrementadas ${ }^{26}$.

Para Serge Latouche, el decrecimiento se sustenta en ocho principios que dan sentido a una acción de resistencia y propuesta frente a un mundo de recursos finitos colonizado por un modelo productivo que promueve la ficción del crecimiento infinito. Los principios consistirían en revaluar, reconceptualizar, reestructurar, redistribuir, relocalizar, reducir, reutilizar y reciclar ${ }^{27}$. Tal y como afirma Clive Steven en El fetiche del crecimiento, el dilema al que nos enfrentamos es fomentar una sociedad materialmente rica e infeliz o iniciar el cambio hacia una más austera pero también más plena ${ }^{28}$. Se trataría en pocas palabras de diseñar una sociedad donde se eliminaran los dogmas que sustentan el capitalismo. El consumo compulsivo y la explotación irreflexiva de la naturaleza, serían liquidados en beneficio de una dinámica centrada en consumir menos, trabajar menos y adoptar un ritmo más pausado, capaz de promover la calidad de la vida social e individual, en vez de rendirse a las demandas del mercado ${ }^{29}$. El decrecimiento busca incrementar la economía del cuidado, o las prácticas desarrolladas por otros pueblos.

El decrecimiento trata de asegurar la sostenibilidad, a la vez que pone de relevancia la insostenibilidad de la propia idea de crecimiento, eje de las políticas del capitalismo. Esta visión pone el dedo en la llaga cuando sitúa en sus justos términos el mito del crecimiento sin fin y su promesa de bienestar. Tal y como los partidarios del decrecimiento señalan el incremento de la producción no elimina la pobreza. El decrecimiento sitúa a las sociedades opulentas del norte ante el desafío de decrecer para ser más felices y mantener el equilibrio perdido con la naturaleza.

En la idea del decrecimiento se pueden observar algunas insuficiencias que la limitan como alternativa viable, aunque sirvan para poner de relevancia la insostenibilidad estructural entre capitalismo y medioambiente. La primera es que el problema fundamental, y sobre el que nos debemos centrar, es la producción que debe ser democratizada, de acuerdo a unos parámetros de utilidad social, que englobe a todas las sociedades. No se puede establecer una diferencia entre sociedades opulentas y pobres. Por muy razonables que seamos con el medio ambiente en los Estados europeos, hay que asumir que representamos una mínima parte del mundo y que el capitalismo tiende a aprovechar las laxas legislaciones medioambientales de muchos Estados para desarrollar su acción. Un ejemplo de ello es China, tanto como productor, como voraz consumidor de recursos naturales, como la energía. Otro elemento a tener en cuenta es que el mundo opulento también se puede dar en determinadas zonas de África o Asia. Allí también hay ricos y áreas comparables con la más rica zona de América o Europa. El capitalismo necesita del crecimiento, pero pensar que por el mero decrecimiento en los Estados opulentos va a contrarrestar la influencia del capitalismo puede conducirnos al error de admitir implícitamen- 
te que el capitalismo produce abundancia, cuando esto no es estrictamente así. Genera grandes diferencias y abundancia tan sólo para unos pocos. El elemento clave no es la cantidad, el cuánto, sino el cómo aparejado a la idea de justicia social.

Según la distinción de Redclift, los límites interiores -0 imperativos sociales y políticos detrás de la acción ambientalestán determinados por el sistema socioeconómico en que vivimos. Los límites exteriores -0 riquezas de la Tierra- pueden modificarse con innovaciones biotecnológicas ${ }^{30}$. En la división entre empobrecidos y enriquecidos, los primeros imponen excesivas tensiones a la conservación medioambiental como consecuencia de las demandas estructurales que se les imponen ${ }^{31}$. Esta división va más allá de la clásica diferenciación entre países o áreas del planeta, generando diferencias de todo orden, nivel y espacio que implican que tengamos que centrarnos en la redistribución de lo que se produce y en cómo se produce, más que en la cantidad de lo producido.

Los instrumentos teóricos que aportan al estudio de los efectos de la producción en el medio ambiente, tanto desde el keynesianismo como desde la economía neoclásica, son incapaces de evaluar la articulación entre la sociedad y el medioambiente. Los efectos sobre el medioambiente son sólo procesos externos a sus paradigmas conceptuales. Al desconocer la lucha de clases como determinante en la reproducción social ignoran la diferencia específica entre los procesos productores de valores de uso y los procesos productivos de valores de cambio y de plusvalía ${ }^{32}$. Por decirlo de otra forma: el coste sobre la Tierra y sobre el futuro quedan fuera del análisis que se realiza desde ambas aproximaciones $^{33}$.

Decrecer pudiera ser más bien un efecto de una transformación productiva y social que haga realmente sostenible la relación entre ser humano y naturaleza, pero no una causa para ello. En este sentido, considero que debemos apostar por una alternativa realista que considere como puntos centrales las condiciones materiales que afectan a los seres humanos y los equilibrios que se han de mantener con la naturaleza. Las esferas de ecología y capitalismo se oponen entre sí. Sólo el cambio social puede lograr una relación más sostenible con el medio ambiente ${ }^{34}$. Preservar estos equilibrios equivale necesariamente a formular modelos productivos alternativos al capitalismo, ya que el fin prioritario no puede ser la maximización del beneficio. A esta alternativa la denominamos sostenibilidad materialista

\section{Sostenibilidad materialista. Hacia un enfoque democrático de la ecología}

Una vez vistas las insuficiencias de las anteriores, podemos concluir que el capitalismo hace imposible conjugar producción con sostenibilidad porque la lógica de la maximización del beneficio impera sobre cualquier otra idea. La lógica expansionista del sistema capitalista hace de la acumulación de riqueza 
en la forma de capital el fin supremo de la sociedad. Rara vez se tiene en cuenta los costes sociales o externos. Incluso los buenos capitalistas que pretende rendir cuentas a la sociedad de sus actividades, al final se ven compelidos en su actuación por sus ineludibles obligaciones con inversores o bancos ${ }^{35}$.

Amputar esta lógica implicaría la muerte del mercado capitalista neoliberal, algo, que es completamente rechazado. No es realista en ninguna forma, decir que se puede limitar la lógica del máximo beneficio en función de la sostenibilidad, ni a nivel teórico ni práctico. Los gobiernos, que deberían proteger los intereses de los ciudadanos, se han puesto a defender los balances de las corporaciones. Estas cuentas de resultados son ajenas a los intereses de los ciudadanos y del medio ambiente.

Antes se fabricaban los productos para que duraran. Un gran número de empresas competían y se jugaban su prestigio por la durabilidad de sus productos y su calidad. Hoy en día, esto cada vez es menos cierto. Los productos son cada vez de peor calidad. El fin no es que una herramienta dure o un mueble pase de generación en generación, sino que sea sustituido al cabo de poco tiempo. Los computadores y equipos electrónicos son un buen ejemplo de esta dinámica del usar y tirar. El plazo de obsolescencia de equipos tecnológicos es cada vez menor. Ni hablar del caso de los teléfonos móviles. Las mismas empresas de telefonía estimulan al consumidor para que cambien de móvil una vez al año. Todos sabemos que estos bienes son muy contaminantes y que la extracción de los minerales, imprescindibles para su fabricación, cuesta la vida de cientos de personas en África, muchas de ellas, niños. Los que podrían poner en prácticas políticas sostenibles no lo hacen por la lógica del mercado y el $80 \%$ restante de la humanidad está demasiado ocupada sobreviviendo para pensar en ello.

\section{Sostenibilidad materialista}

John Bellamy Foster es quizá uno de los autores que más y mejor han estudiado la preocupación ecológica de Marx. Bellamy Foster considera que el concepto de fractura metabólica que Marx acuña para designar los efectos del sistema capitalista sobre la interacción metabólica entre naturaleza y sociedad, supone que el sistema capitalista, con la división del trabajo y el antagonismo entre ciudad y campo sobre los que se va desarrollando, genera la alienación más básica y fundamental del ser humano con respecto a la tierra que es "condición sine qua non del sistema capitalista» y que, a su vez, fractura o interrumpe el metabolismo de sociedad y naturaleza ${ }^{36}$.

Bellamy Foster resalta como el propio Marx consideraba que un trato consciente y racional de la tierra como propiedad comunal permanente es la condición inalienable para la existencia y reproducción de la cadena de las generaciones humanas». Según Bellamy Foster, para Marx «la revolución contra el capitalismo requería tanto terminar con sus específicas relaciones de explotación del trabajo, como -a través de la 
regulación racional de las relaciones metabólicas entre los seres humanos y la naturaleza por medio de la ciencia y la industria modernas- trascender la alienación con respecto a la tierra: el último fundamento/condición previa del capitalismo. Solamente en estos términos tiene sentido el frecuente llamamiento de Marx a la «abolición del trabajo asalariado». ${ }^{37}$

Estamos situados en una sociedad de "usar y tirar». Producimos toneladas de basura y todos los expertos coinciden en que el capitalismo necesita varios planetas para mantener este ritmo frenético. Este modelo productivo es lo antagónico de lo sostenible. Lo sostenible estaría en una lógica de la responsabilidad y el modelo actual se inserta en una lógica de la maximización del beneficio.

En la sociedad de usar y tirar los ciudadanos se convierten en meros consumidores. Este es el espacio de acción social que se les ha reservado. Pueden decidir y elegir lo que comprar. Ciudadanos se transmutan en consumidores y la democracia queda supeditada a la maximización del beneficio. El ciudadano es expulsado de la política y se asume que el mercado es eficiente para asignar bienes y para hacerle feliz.

El mercado capitalista es un mito. Un mito con extraordinaria potencia. Todo sus fundamentos mitológicos no son hoy otra cosa que ensoñaciones. La libre competencia significa mayor concentración empresarial y erosión de las normas antitrust; asignación eficiente de bienes y servicios, quiere decir privatizar y encarecer la vida de las endeuda- das familias. Al final ha tenido que ser el dinero de los contribuyentes el que salvara de la quiebra a grandes bancos y empresas. ¿Es eso eficacia? Como señala John Lanchaster, esta economía utópica, asentada en el quebranto del sentido común, en la abstracción y el ensimismamiento, ha hundido el mundo en una crisis económica terrible ${ }^{38}$. No es el primero, pero si uno de los últimos que ponen de manifiesto que nos enfrentamos a un dogma sustentado en una racionalidad irracional. Es irracional porque compromete el planeta y a la mayoría de los que en él viven. Es «racional» porque beneficia a corto plazo $y$, de forma extraordinaria, a unos pocos. En consecuencia, la sostenibilidad es lo que desconocemos.

La sostenibilidad es una reacción ante la regla general, el consumismo; una forma de alterar el triángulo hegemónico formado por producción antisostenible, lógica del máximo beneficio y mercado. El modelo de relaciones productivas hegemónico es eficiente para lograr objetivos neoliberales, pero incapacitado para la sostenibilidad. En el siguiente epígrafe analizaremos las razones que conducen a esta afirmación y esbozaré posibles alternativas.

\section{Desarrollo y ecología}

El proceso cultural capitalista ha concebido la naturaleza como un objeto del que apropiarse y al que explotar. Hoy en día se concibe la naturaleza como un objeto que se puede patentar y del que podemos excluir a los otros en su disfrute. Ejemplo de ello es la biopira- 
tería, es decir, el consciente robo de conocimientos tradicionales a pueblos indígenas, que generosamente confían su saber a corporaciones que los usarán en beneficios de unos pocos ${ }^{39}$. Tragedias como el suicidio de decenas de personas de los pueblos indígenas de Brasil (por ejemplo los Sabante o los Carowa en Mato-Groso, por causa de la soja) ilustran estas prácticas.

Nos encontramos ante un problema de pensamiento. En una representación colectiva obcecada en la consecución de los valores que fundan el imaginario capitalista en todas sus vertientes. Un imaginario que cifra sus expectativas en el máximo beneficio, en el consumismo y en la cuenta de resultados. Un dogma que ignora el entorno inmediato en beneficio de un futuro prometedor, asentado en un mito, en una promesa constantemente incumplida. Los ajustes estructurales habidos en América Latina en los 90 prometían un futuro maravilloso a cambio de sacrificios. Hoy en 2011 sabemos cuál es el resultado: más emigración y más concentración de la riqueza.

Dubos señaló que en las sociedades industriales se tiende a ignorar el entorno inmediato, en beneficio de objetivos lejanos $^{40}$. De esta manera, se perpetúa la idea de que siempre hay un paraíso al que tender y por el que vale la pena hacer los esfuerzos y sacrificios necesarios. Frente a esta situación, para Jonas existe un imperativo ético nuevo, no recogido en el pasado, que dice al ser humano que debe obrar de tal manera que los efectos de su acción sean compatibles con la supervivencia de la vida en el planeta Tierra ${ }^{41}$. Es una ética ecológica que se hizo eco teórico de las propuestas políticas realizadas por los nuevos movimientos sociales de su tiempo. Posteriormente, Hinkelammert, propuso interesantes críticas a las notables insuficiencias del modelo weberiano de la eficiencia y la calculabilidad abstracta que presupone un conocimiento perfecto de la realidad (el ejemplo del que se vale es la eficiencia de aquellos que sierran la rama en la que se encuentran sentados) y el determinismo derivado del mismo. Hinkelammert defiende lo que denomina una lógica de la vida capaz de mantener el equilibrio ecológico sobre la Tierra ${ }^{42}$.

Trabajos como los de Jonas o Hinkelammert son básicos en cualquier discusión sobre la relación entre el ser humano y la naturaleza. Durante los siglos de formación del pensamiento occidental los problemas medioambien-tales fueron ignorados, ya que éstos no se presentaban, ni remotamente, con la agudeza del presente. Tal vez, tal ignorancia no sea sólo consecuencia de la ausencia del problema, sino del propio pensamiento. Un pensamiento que no contempla la necesidad del equilibrio ecológico como una prioridad. Siguiendo a David Bohm: «imaginemos un arroyo que haya sido contaminado cerca de su nacimiento y quienes viven río bajo, ignorantes de lo que ocurre, intenten eliminar la contaminación depurando el agua. Pero el hecho es que su tentativa bien pudiera ocasionar más contaminación, porque la acción correcta sería la de considerar el río en su totalidad y abordar el problema desde su origen. Del mismo modo, en mi opinión, es el origen mismo del pensamiento el que se halla contaminado. Uno podría aducir, 
no sin cierta razón, que el error se originó cuando por primera vez se vertieron productos contaminantes al río, pero el hecho, en el caso del pensamiento, es que nosotros seguimos contaminándolo. El origen de esta situación, por tanto, no se halla en el tiempo, no se ubica en algún remoto pasado sino que, por el contrario, se encuentra siempre en el ahora» ${ }^{43}$. El hombre de la sociedad industrial se considera al mismo tiempo un ser que está separado de la naturaleza y atascado en la cultura que él mismo se ha creado. Desgastado y frustrado aparece como el guardián obstinado de su propia prisión ${ }^{44}$.

La vida en sociedad es conceptuada como una competición tecnológica y económica con el fin de obtener un progreso material ilimitado, en vez de una comunidad democrática donde se avanza entre contrastes cualitativos y prácticas sociales innovadoras ${ }^{45}$. Según Morin, la creatividad humana se ha puesto en hibernación en sociedades fosilizadas; pero ésta conciencia puede despertar en tiempos de crisis como los actuales. Todo precisa ser transformado ${ }^{46}$.

Es necesario, una revisión radical del marco teórico en el que hemos estado y estamos sumidos. Esta revisión se trató de llevar a cabo en 1992 con la Cumbre de la Tierra en Río de Janeiro, donde se alumbró el concepto de desarrollo sostenible. Es una concepción matizada de la tradicional relación del modelo hegemónico económico. En poco tiempo se ha hecho con una gran popularidad e incluso prestigio social. Al mismo tiempo, poca gente tiene una idea clara de lo que significa. Se sabe que es el resultado de una toma de conciencia de la degradación que sufre la Tierra. Se trata de buscar un equilibrio entre desarrollo y protección del medio ambiente. John Bellamy Foster, estima que el desarrollo sostenible es un concepto económico que ofrece perseverar en el modelo económico actual, pero con unas correcciones que posibiliten la protección de la naturaleza. Esto lo hace ineficiente para los fines que se propone el propio desarrollo sostenible ${ }^{47}$. Ambas lógicas no pueden convivir. Bellamy Foster estima que la lucha por crear un mundo más verde está vinculada a la lucha por reducir la injusticia social48. Para una nueva lógica de relación con la naturaleza es imprescindible alterar la lógica económica. Como señala Lowy y Kovel en su Manifiesto Ecosocialista, la democratización económica -que implica la socialización de las fuerzas productivasconduce a que las grandes decisiones sobre la producción y la distribución no serán tomadas por «los mercados» o por un politburó, sino por la sociedad misma después de un debate democrático y pluralista en el cual se opongan las propuestas y las opciones distintas ${ }^{49}$.

Como afirmé en el punto anterior, el capitalismo se basa en la acumulación y en la maximización del beneficio. Éstas son las dos prioridades. No la protección de la naturaleza. Incrementar la producción no evita la pobreza. Los seres humanos son parte de la naturaleza. La explotación de la naturaleza forma parte de la explotación de unas personas por otras. La degradación de la naturaleza alcanza a los seres humanos y sus relaciones. La ciudadanía queda reducida a lo que Paulo Freire denominó «cultura del silencio». Una situación 
producto de la pobreza, la desesperanza y la incapacidad de tomar decisiones sobre su futuro ${ }^{50}$. La lucha ecologista es inseparable de la lucha por la justicia y contra el capitalismo. Hay que buscar un desarrollo humano, que permita el bienestar y la calidad de vida de los seres humanos. Lo suficiente, frente a la acumulación y el exceso. Protocolos como el de Kioto son una contribución claramente insuficiente, ya que mantiene el sistema productivo que hace de la insostenibilidad la propia razón de su existencia. Es necesario ofrecer un modelo de desarrollo alternativo; crítico con el capitalismo y basado en un desarrollo humano cuyo fin sea lograr una producción dirigida a cubrir las necesidades de la sociedad y el equilibrio de la naturaleza.

Riechmann señala seis principios básicos para la biomímesis que son de gran utilidad para la construcción de una sostenibilidad materialista y que el autor de Barcelona conecta con una reconstrucción ecológica de la economía:(†a) Estado estacionario; b) Vivir del Sol; c) Cerrar los ciclos de materiales; d) No transportar demasiado lejos los materiales; e) Evitar xenobióticos; f) Respetar la diversidad. En definitiva, desarrollar ciclos de materiales cerrados, sin contaminación y sin toxicidad, mo- vidos por energía solar, adaptados a la diversidad local: ésta es la esencia de una economía sostenible ${ }^{51}$. Pero nada de ello es aceptable desde el capitalismo. Es inviable imponer este tipo de consideraciones a la lógica de la maximización e beneficio.

Una sostenibilidad materialista en definitiva estaría basada en la negación del capitalismo como modelo productivo y cultural y la reivindicación de una producción democrática y participada, en el marco de una ética capaz de conciliar los intereses de los seres humanos y de la Tierra. La naturaleza no es un objeto que explotar, sino un componente más de nuestra singularidad, superadora de cualquier tentación individualista o aislacionista. La sostenibilidad materialista se conformaría sobre las bases de la perdición del ser humano y el abandono de perniciosos idealismos que prometen al ser humano un crecimiento indefinido.

Las posiciones que hemos estudiado pueden ser resumidas de la siguiente forma:

Podemos resumir los tres enfoques mencionados en tres rombos que representarían esquemáticamente cada visión:

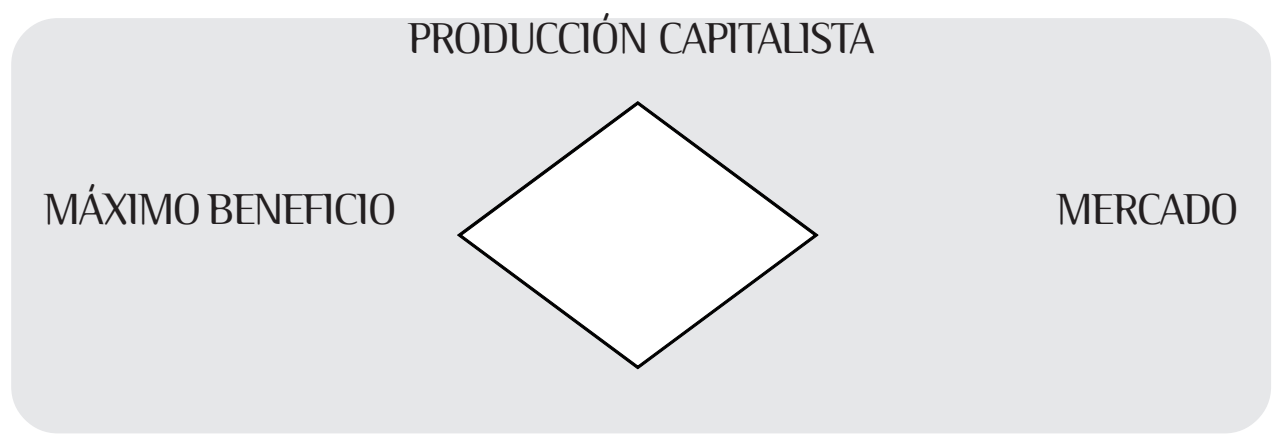

Revista Internacional de Pensamiento Político - I Época - Vol. 6 - 2011 - [395-418] - ISSN 1885-589X 


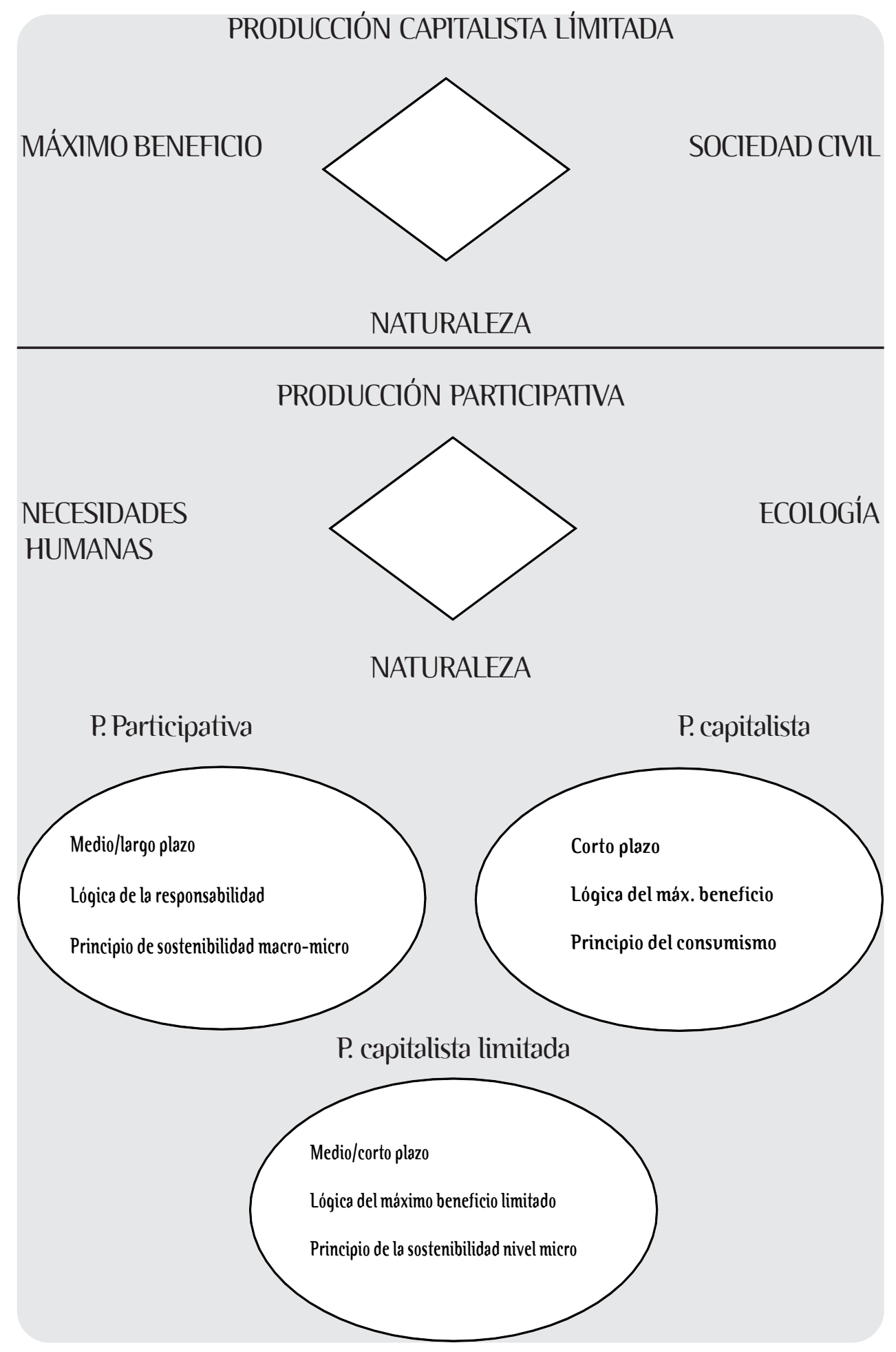


Libertad y responsabilidad son partes de la misma matriz. La naturaleza y el ser humano merecen profundizar en modelos de desarrollo diferentes al hegemónico, que sitúen las necesidades de los seres humanos y la justicia social como fin prioritario. Todos somos responsables de nuestro futuro y nada ni nadie ha de decidir por nosotros, ya que en eso nos jugamos, tanto el desarrollo de nuestra personalidad como seres humanos, como el de nuestras sociedades y las posibilidades de vida para las generaciones futuras en el planeta Tierra.

\section{Conclusiones}

El 11 de marzo de 2011 un acontecimiento horrorizó al mundo. El terremoto y tsunami de Japón provocó miles de muertos, heridos y personas sin hogar. Y no sólo eso: se produjo una de las peores crisis nucleares de la historia. El intento de muchos medios de comunicación y de gobiernos por relativizar el gravísimo accidente nuclear aún nos provoca sonrojo e indignación. En los días posteriores, muchos ejecutivos de entidades financieras aprovecharon la muerte y desolación para hacer negocio en las bolsas del mundo. ¿Se puede calificar como humano a un sistema así?

Carl Amery, fundador de Los Verdes en Alemania, publicó hace unos años un trabajo titulado Auschwitz, ¿comienza el siglo XXI? En este libro Amery considraba errada la tendencia generalizada de interpretar el periodo del Tercer Reich como una excepcionalidad histórica. Muy al contrario: para Amery existe no sólo continuidad con la historia anterior al auge de Hitler, sino, lo que es aún peor, pervivencia en el seno de la sociedad contemporánea. Según Amery la crisis de la biosfera es sobre todo una crisis cultural que sitúa a un modelo económico aliado del desierto, como el patrón al que todo se subordina; el mundo de la economía y las finanzas no sólo se ha liberado de la responsabilidad biosférica, sino también de la responsabilidad humana, orbitando en torno al Sol de los beneficios ${ }^{52}$.

La sostenibilidad no puede mantenerse en el modelo capitalista neoliberal actual. Es imposible hacer compatible un modelo productivo cuyas bases son el máximo beneficio y un mercado dominado por las grandes corporaciones. Es un error continuar la ineficiente estrategia actual. Esta estrategia consiste en incluir la idea de sostenibilidad en todo el aparato social, cultural e ideológico del neoliberalismo.

El coral en Indonesia se muere, la deforestación en el Amazonas continúa y las víctimas de ello no sólo es la naturaleza, sino las personas que obtienen su sustento de ello. Todo está interconectado y los problemas ecológicos, lo son también políticos, económicos y culturales. No podemos lamentarnos por los efectos de un modelo socioeconómico insostenible, si permitimos que las dinámicas que los causan permanezcan inalteradas.

Se continúa con una visión parcial que da un tremendo poder a la Organización Mundial del Comercio -WTO-y a las corporaciones, mientras se hacen galas benéficas a favor de las focas o contra el cambio climático. Si queremos lograr 
la sostenibilidad, debe alterarse la manera en la que producimos y en la que concebimos ideas, valores y principios.

Las alternativas existen. Se han puesto en práctica en el espacio local. Desde una sostenibilidad materialista es imprescindible apostar por una democratización en las relaciones de producción que necesariamente tendrán consecuencias en el medioambiente. La clave es cambiar las prioridades. Una nueva hegemonía, capaz de situar la producción democrática, necesidades humanas y ecología como dominantes facilitará una nueva relación entre el ser humano y la naturaleza antes de que sea demasiado tarde para cambiar. Esa estrategia costará tiempo y sacrificio, puesto que estamos en la dirección contra- ria. Si no lo remediamos, podemos estar en los albores de un neototalitarismo brutal que cada día condena más a nuestro planeta y a los seres humanos.

Democracia y sostenibilidad están indisolublemente unidas. Sólo si los intereses de la mayoría se salvaguardan estaremos cerca de la sostenibilidad. Un solo país, una sola región del mundo no lo puede hacer. Debe ser algo que incluya a la mayoría de la población o de lo contrario no será funcional. Cuando la minoría es la que decide, la sosteni-/ bilidad no es la prioridad y lo importante es ganar dinero. Pero si la mayoría toma las decisiones puede que esto cambie y quizás no hagamos un mundo perfecto, sino algo mejor que lo conocido.

\section{Notas}

1<http://www.elpais.com/articulo/sociedad/ ciudadano/7000/millones/elpepisoc/ 20110926elpepisoc_1/Tes>.

2 Donatien Garnier, La muerte prefiere a los pobres, en VV.AA., Anuario económico geopolítico mundial «El estado del mundo 2011, Akal, Madrid, 2011, pp. 172-173.

${ }^{3}$ Ernst Fritz Schumacher, Lo Pequeño es hermoso, Blume, Barcelona, 1978.

${ }^{4}$ Los mercados exigen a los países en dificultades crediticias que tomen medidas impopulares (recortes de gasto público, principalmente) con el fin de calmar su incertidumbre crediticia. Es lo que está sucediendo a países como Irlanda, Portugal o España. Las agencias de calificación de riesgo tienen unos objetivos que poco tienen que ver con la protección medioambiental y, sin embargo, ejercen una influencia decisiva en las políticas económicas de los Estados que tienen consecuencias en la protección de la naturaleza.

${ }^{5}$ En este sentido, Jean Claude Junker manifestó con motivo del plan de ajuste de Grecia en junio de 2011, que este país había perdido parte de su soberanía.

${ }^{6}$ Como señala Jorge Riechmann, la palabra ecomímesis se acuñó, a mediados de los años noventa (Gil Friend, "Ecomimesis: copying ecosystems for fun and profit», The New Bottom Line, 14 de febrero de 1996). Citado por Riechmann en Jorge Riechmann, «Biomímesis. Respuestas a algunas objeciones», Argumentos de razón, 9, 2006.

${ }^{7}$ Según ha reconocido recientemente la IOM (International Organization for Inmigration) con sede en Ginebra en su conferencia sobre Cambio climático, degradación ambiental y migración de 29-30 de marzo de 2011, la realidad del cambio climático complica considerablemente el nexo entre el medio ambiente y la migración. Los factores medioambientales, entre ellos los efectos del cambio climático, que abarcan los desastres naturales o la degradación ambiental tienen repercusiones negativas en países, ciudades y poblaciones de distintas partes del mundo 
que están particularmente expuestas y son especialmente vulnerables. V. <http://www. pnuma.org/forumofministers/15-venezuela/ ven13tre-EcosistemasdelMilenioEsp.pdf>.

${ }^{8}$ Los resultados de la reciente Cumbre de Cancún de diciembre de 2010 han sido más que discretos. Sólo la disposición general de salvar la cara frente a la opinión pública pudo poner de acuerdo a los Estados en ciertos acuerdos, claramente insuficientes para la protección medioambiental. Este modelo de cumbres (como antes fue Copenhaguen o Kioto) es un procedimiento fracasado para la gestión de estos problemas. La no presencia decisiva de corporaciones -principales productores de problemas medioambientales- o la indiferencia de China o EE.UU. la convierten en un ejercicio de relaciones públicas, más que una herramienta útil de preservación de la naturaleza.

${ }^{9}$ Edgar Morin, uno de los expertos mundiales en metodología y padre del pensamiento complejo, señala que es imprescindible acabar con el pensamiento simplista que establece que los saberes deben situarse en compartimentos estancos sin conexión los unos con los otros. Para Morin, sólo un pensamiento que tenga en cuenta la complejidad de la realidad y la conexión e implicación que existe entre los desafíos que tenemos planteados, podrá servir para establecer estrategias y acciones que ayuden a la supervivencia del planeta (Edgar Morin y Anne B. Kern, Tierra Patria, Kairós, Barcelona, 1994, pp. 26 y ss.)

${ }^{10}$ Antonio Escohotado, Orden y caos, Espasa, Madrid, 1999, p. 127.

11 Fritjof Capra, El Punto Crucial. Ciencia, sociedad y cultura naciente, Integral, BarceIona, 1985, p. 107.

12 Alfonso Pérez-Argote, Medio ambiente e ideología en el capitalismo avanzado, Encuentro, Madrid, 1979, p. 193.

13 Id., p. 208.

14 Karel Mayrand y Sylvie Trottier, Investigación sobre el ecoblanqueamiento, en VV. AA., Anuario económico geopolítico mundial «El estado del mundo 2011, op. cit., p. 175. Las últimas investigaciones indican que la mer- cadotecnia ecológica está siendo muy nociva para la difusión de valores relacionados con la sostenibilidad ambiental. Son especialmente mal recibidos aquellos mensajes que proceden de empresas y gobiernos.

15 V. Jorge Riechmann, Un mundo vulnerable, Los Libros de la Catarata, Madrid, 2000. Riechmann ha formulado tres formas de insertar este principio. 1. Conferencias de consenso: tras recibir informaciones de los expertos los ciudadanos deliberan y emiten un informe en que manifiestan sus recomendaciones. 2. Talleres de escenarios: Ios participantes de distintos intereses se reúnen para analizar los distintos escenarios posibles presentados por los interesados y proponer el procedimiento más adecuado para la acción. 3. Talleres de ciencia: los ciudadanos se reúnen en centros independientes, como universidades o instituciones sin ánimo de lucro, que les ayudan a llevar a cabo determinada investigación para ofrecer una respuesta adecuada a la gestión del riesgo (Jorge Riechman, «Introducción: un Principio para reorientar relaciones de la humanidad con la biosfera», en Jorge Riechmann, Joel Tickner (coord.). El Principio de precaución. En medio ambiente y la salud pública: de las definiciones a la práctica, Icaria, Barcelona, 2002, pp. 66-67).

16 Bjorn Lomborg, El Ecologista Escéptico, Espasa, Madrid, 2003, p. 74.

17 En este sentido, son muy reveladoras las estadísticas publicadas en El estado del mundo 2011 (VV.AA., op. cit., pp. 618-625). Una de las estadísticas relativas a nuestro tema que podemos subrayar es el crecimiento en consumo de combustibles fósiles, especialmente demandados por las economías en ascenso. A la vez llama la atención el nivel de los precios de alimentos básicos como cereales en los Estado en desarrollo. Estos precios siguen por encima de los niveles registrados en la crisis de 2008, lo que augura emergencias alimentarias en las zonas más pobres del planeta en los próximos años (Fuente: Enerdata, junio de 2010).

18 Douglas Torgerson «Constituting green democracy: A political project.» The Good Society, 17, 2, 2008, pp. 18-24. Para Tor- 
gerson es imprescindible desarrollar la idea de una sociedad democrática frente a la democracia como forma de gobierno. De esta forma, se podrían desarrollar los objetivos cooperativos y conservacionistas de la democracia verde.

19 Nicolás Martín Sosa, «A vueltas con la sustentabilidad, esta vez desde la ética», Sistema,162-163, junio 2001, pp. 53-72. V. Marisa Ibáñez, «Nicolás Martín Sosa. Un Pionero dela ética Ecológica en España», Isegoría, 32, 2005, pp. 297-299.

20 John Dryzek, "Political and Ecological Communication», Environmental Politics, 4, 4, 1995, pp. 23, 28-29.

21 Giles Slade, Made to Break: Technology and Obsolescence in America, Harvard Univ. Press, Cambridge, 2006, pp. 4 y ss.

22 Andrew G. Kirk, Counterculture Green, The Whole Herat Catalog and American Environmentalism, University Press of Kansas, Lawrence, 2007, pp. 206-207.

23 Paul Hawken, The Ecology of Commerce, HarperCollins, New York, 1993, pp. 55-56.

24 Facpe, "Los productos viajeros, El impacto de los grandes supermercados», 5, Primavera-Verano, 2010.

25 <http://www.sevilladecrece.net/>.

26 V. Carlos Taibo, En defensa del decrecimiento. Sobre capitalismo, crisis y barbarie, Los Libros de la Catarata, Madrid, 2009.

27 Serge Latouche, S. (2009), Pequeño tratado del decrecimiento sereno, Icaria, Brcelona, pp. 14 y ss.

${ }^{28} \mathrm{~V}$. Clive Hamilton, El fetiche del crecimiento, Laetoli, Pamplona, 2006, pp. 33-39.

29 Id.

${ }^{30}$ En este sentido es interesante que subrayemos la promesa incumplida de las grandes corporaciones de acabar con el hambre en el mundo gracias a los transgénicos. Toda esa retórica se ha revelado como simplemente justificativa de acciones encaminadas a monopolizar el mercado agrícola y evitar que los agricultores usen sus propias semillas, como tradicionalmente han hecho. La biotecnología y sus avances pueden ayudar a mejorar la relación de los seres humanos con la naturaleza, pero cuando ésta tecnología se enfoca a la maximización del beneficio, se invalida cualquier posibilidad de su uso para el equilibrio medioambiental. V. Daniel Charles, Lords of the Harvest. Biotech, Big Money, and The Future of The Food, Basic Books, New York, 2001, pp. 200 y ss. En esta parte del libro se analiza la agresiva política de Monsanto y sus efectos sobre el medioambiente.

${ }^{31}$ Michael, Redclift, Los conflictos del desarrollo y la crisis ambiental, FCE, México, 1984, pp. 232-233.

32 Enrique Leff, Ecología y capital. Hacia una perspectiva ambiental en el desarrollo, UNAM, México, 1986, pp. 31-32.

33 David Brower, It's Healing Time on Earth, en Hildegarde Hannum (ed.), People, Land and Community, Yale University Press, New Haven, 1997, p. 294.

34 John Bellamy Foster, Ecology Against Capitalism, Monthly Review Press, New York, 2002, p. 7.

35 Id., p. 9 y 12.

36 John Bellamy Foster, La ecología de Marx. Materialismo y naturaleza, El Viejo Topo, Barcelona, 2000, pp. 268, 272.

$37 \mathrm{Id}$.

38 John Lanchester,jHuy!: Por qué el mundo debe a todo el mundo y nadie puede pagar, Anagrama, Barcelona, 2010, p. 4.

39 Vandana Shiva, Biopiratería: El saqueo de la naturaleza y del conocimiento, Icaria, Barcelona, 2001, p. 4.

40 Rene Dubos, Un Dios interior, Salvat, Barcelona, 1986, p. 91.

41 Hans Jonas, El principio de responsabilidad. Ensayo de una ética para la civilización tecnológica, Herder, Barcelona, 1995, p. 9.

42 Franz Hinkelammert, El mapa del emperador. Determinismo, caos y sujeto, DEI, San José, 1996, pp. 14 y ss.

43 David Bohm Sobre el diálogo, Kairós, Barcelona, 1997, pp. 85-86.

44 Dominique Simonet, El Ecologismo, Gedisa, Barcelona, 1980, pp. 101-106. 
${ }^{45}$ Charles Taylor Sources of the self. The Making of the Modern Identity, Harvard University Press, Cambridge 1992, p. 112; Edgar Morin y Anne B. Kern, Tierra-Patria, Kairós, Barcelona, 1993, p. 47.

46 Etre, Laurent, Quel retour à Marx pour penser notre temps? Debats. L'Humanité, 17 avril 2010.

47 John Bellamy Foster, Ecology Against Capitalism, Monthly Review Press, New York, 2002, p. 79.

48 Id., pp. 80-81.
49 Michael Lowy Joel Kovel, An Ecosocialist Manifesto, en <http://www.ecosocialist network.org/Docs/EcoManifesto.htm>.

50 HillaryWainwright, Reclaim the State. Experiments in Popular Democracy, Verso, New York, 2003, 12 y ss.

51 V. Jorge Riechmann, "Biomímesis. Respuestas a algunas objeciones», Argumentos de razón, 9, 2006.

52 Carl Amery, Auschwitz, ¿comienza el siglo XXI? Hitler como precursor, Turner FCE, Madrid, 2002, pp. 160-161. 